Document downloaded from:

http://hdl.handle.net/10251/82905

This paper must be cited as:

Morillas, S.; Grecova, S. (2016). Perceptual similarity between color images using fuzzy metrics. Journal of Visual Communication and Image Representation. 34:230-235. doi:10.1016/j.jvcir.2015.04.003.

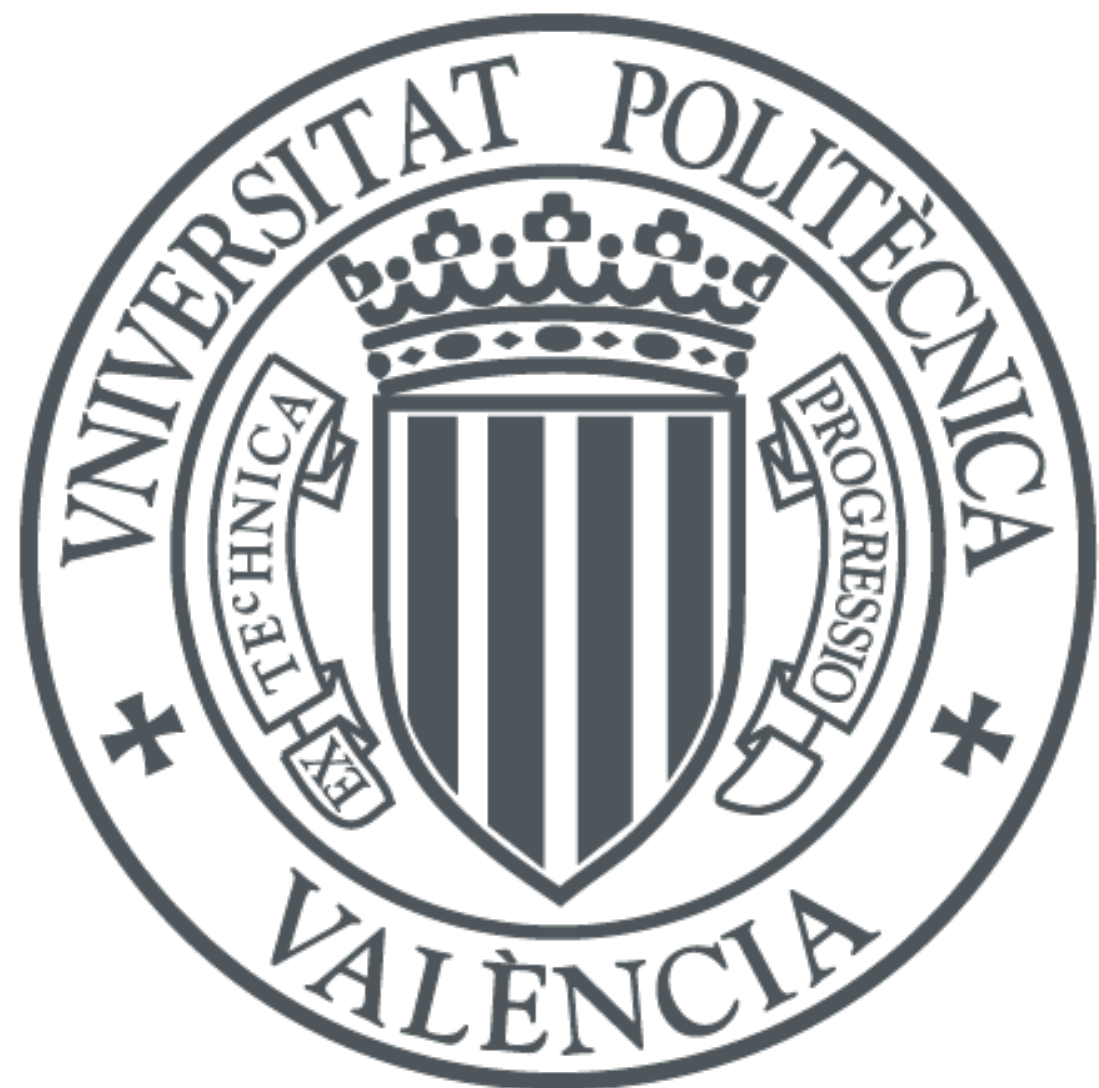

The final publication is available at

https://doi.org/10.1016/j.jvcir.2015.04.003

Copyright Elsevier

Additional Information

"NOTICE: this is the author's version of a work that was accepted for publication in Journal of Visual Communication and Image Representation. Changes resulting from the publishing process, such as peer review, editing, corrections, structural formatting, and other quality control mechanisms may not be reflected in this document. Changes may have been made to this work since it was submitted for publication. A definitive version was subsequently published in Journal of Visual Communication and Image Representation, [Volume 34, January 2016, Pages 230-235] https://doi.org/10.1016/j.jvcir.2015.04.003 


\title{
Perceptual similarity between color images using fuzzy metrics
}

\author{
Svetlana Komara ${ }^{\mathrm{a}, *}$, Samuel Morillas ${ }^{\mathrm{b}}$ \\ ${ }^{a}$ Department of Mathematics, University of Latvia, Latvia, komara.svetlana@gmail.com \\ ${ }^{b}$ Instituto Universitario de Matemática Pura y Aplicada, Universidad Politécnica de \\ Valencia, Spain,smorillas@mat.upv.es
}

\begin{abstract}
In many applications of the computer vision field measuring the similarity between (color) images is of paramount importance. However, the commonly used pixelwise similarity measures such as Mean Absolute Error, Peak Signal to Noise Ratio, Mean Squared Error or Normalized Color Difference do not match well with perceptual similarity. Recently, it has been proposed a method for gray-scale image similarity that correlates quite well with the perceptual similarity and it has been extended to color images. In this paper we use the basic ideas in this recent work to propose an alternative method based on fuzzy metrics for perceptual color image similarity. Experimental results employing a survey of observations show that the global performance of our proposal is competitive with best state of the art methods and that it shows some advantages in performance for images with low correlation among some image channels.
\end{abstract}

\footnotetext{
*Corresponding author. Tel.: +37129799678; complete postal address: Reznas 10 k-1, Riga, Latvia, LV-1019.
}

Preprint submitted to Journal of Visual Communication and Image RepresentationFebruary 13, 2015 
Keywords: Color Imaging, Fuzzy Logic, Fuzzy Metrics, Perceptual Image Similarity

\section{Introduction}

Many applications in the fields of image processing and computer vision use image similarity measures for different purposes [1]. In some cases the objective is the very measurement of the similarity itself globally or partially in the images, but other times the similarity is used to assess the performance of an image processing method. For instance, in image filtering, the common process to measure the performance of a filtering method is the following: an original image is corrupted artificially with noise, then it is filtered with the method under study and it is measured how similar is the filtered image to the original one. This allows to properly adjust filter parameters for optimal performance, to assess different filter configurations as well as to compare the performance of different filtering methods. An analogous approach is used in other image processing procedures such as image compression, image demosaicing or video de-interlacing. Therefore, the similarity measure used highly influences the whole process.

The most common similarity measures used in this context are based on a pixelwise approach, such as the Mean Absolute Error (MAE), the Mean Squared Error (MSE), the Peak Signal to Noise Ratio (PSNR) or the Normalized Color Difference (NCD) (which is the MSE in the Lab color space). However, these measures do not match well with perceptual observations 
and, as the MSE, some of them have other concerns [2].

During the last twenty years series of works have addressed the problem of defining image similarity measures that match human perceptual similarity. First works in this issue include the Weighted Signal to Noise Ratio (WSNR) [3] which simulates the human visual system properties by filtering both the reference and distorted images with contrast sensitivity functions and then compute the SNR. Other measures $[4,5]$ assess shifts in image luminance, differences in the frequency domain and changes in edges. Instead of luminance, some metrics $[6,7,8]$ specifically target color in images. Other metrics $[9,10]$ embed a hidden signal in an image, introduce an impairment and measure its quality. Besides, to detect similarity between images their histograms have been used $[11,12]$.

More recently, in $[13,14]$ a similarity measure for gray-scale images that matches well with perceptual similarity has been introduced (UQIUniversal Quality Index and SSIM-Single-scale Structural Similarity Index). This method could be applied in color images in a componentwise fashion, that is, independently in each color channel and then averaged. However, it is well-known that the correlation among the color image channels should be taken into account and this approach cannot provide optimal performance [1], as we show in this paper. This similarity measure is extended to the Multiscale Structural Similarity Index (MSSIM) in [15]. In turn, in [16], a color comparison criterion is combined with MSSIM. In the approach [17], SSIM scores are weighted by region type. And, in [18], a two staged wavelet based 
Visual Signal to Noise Ratio (VSNR) was defined based on the low-level and the mid-level properties of human vision.

In this paper, we introduce a method for color image similarity that matches perceptual similarity. Our method follows a procedure inspired in $[13,14]$ as follows: the images are processed with sliding patches so that a number of small image portions are compared and the similarity between two images is obtained by averaging the similarities of all portions. In each pair of patches three different factors are compared separately and then combined: contrast, structure and luminance. The particular expressions used in $[13,14]$ for these three factors cannot be directly generalized from gray-scale images to color images, so we propose our own expressions to measure them. Experimental results employing perceptual similarity observations show that our approach is able to outperform classical similarity measures, is competitive with best state-of-the-art methods, and shows some advantages in performance for images with low correlation among some image channels.

In the following section we detail the proposed method. Section 3 contains the experimental results and discussion. Finally, Section 4 presents the conclusions.

\section{Proposed image similarity measure}

Let $\mathbf{X}$ denote a RGB image and $W$ be the sliding patch of finite size $q \times q=n$ used to process the image. The image pixels in $W, \mathbf{X}_{W}$, are denoted as $\mathbf{x}_{i}(l), i=1, \ldots, n$ where $l=1,2,3$ denotes the $\mathrm{R}, \mathrm{G}$, and $\mathrm{B}$ channels, 
respectively. Notice that $\mathbf{x}_{i}$ can be processed as a three component vector.

We measure the similarity between images $\mathbf{X}$ and $\mathbf{Y}$ as the average of the similarities of the image patches $\mathbf{X}_{W}$ and $\mathbf{Y}_{W}$ obtained when sliding the patch along every image row. To measure the similarity between two patches in the same image location we measure three different similarities: contrast, structure and luminance. In so doing, we need to measure the similarities between all image color pixels $\mathbf{x}_{i}$ and $\mathbf{y}_{i}$ in $\mathbf{X}_{W}$ and $\mathbf{Y}_{W}$, respectively, and the mean color vector in each patch, $\overline{\mathbf{x}_{W}}$ and $\overline{\mathbf{y}_{W}}$. We denote these similarities by $M_{\mathbf{x}_{i}}$ and $M_{\mathbf{y}_{i}}$ and we measure them by employing the fuzzy metric used in $[19,20,21,22]$ for its high sensitivity to edges as follows.

$$
M_{\mathbf{x}_{i}}=M\left(\mathbf{x}_{i}, \overline{\mathbf{x}_{W}}, t\right)=\prod_{l=1}^{3} \frac{\min \left(x_{i}(l), \overline{\mathbf{x}_{W}}(l)\right)+t}{\max \left(x_{i}(l), \overline{\mathbf{x}_{W}}(l)\right)+t}, \quad i=1, \ldots, n,
$$

where $t>0$ and

$$
\overline{\mathbf{x}_{W}}=\frac{1}{n} \sum_{j=1}^{n} x_{j}, \quad l=1,2,3
$$

Through an analogous computation in the image $\mathbf{Y}$ we obtained the similarities $M_{\mathbf{y}_{i}}, i=1, \ldots, n$. Notice that $M_{\mathbf{x}_{i}}$ and $M_{\mathbf{y}_{i}}$ are fuzzy similarities that take value in $[0,1]$. 


\subsection{Contrast}

Contrast can be seen as the largest difference observed in $\mathbf{X}_{W}$ and $\mathbf{Y}_{W}$. We can measure contrast in $\mathbf{X}_{W}$ using $M_{\mathbf{x}_{i}}$ as $C_{\mathbf{X}_{W}}=\max \left(M_{\mathbf{x}_{i}}\right)-\min \left(M_{\mathbf{x}_{i}}\right), \quad i=$ $1, \ldots, n$, and analogously for $\mathbf{Y}_{W}$. Then, the fuzzy similarity between the contrasts is given by

$$
S C\left(\mathbf{X}_{W}, \mathbf{Y}_{W}\right)=1-\left|C_{\mathbf{X}_{W}}-C_{\mathbf{Y}_{W}}\right| .
$$

\subsection{Structure}

Structure describes how the differences between the pixels in a patch are distributed spatially. Therefore, for this aspect we average the fuzzy similarities of $M_{\mathbf{x}_{i}}$ and $M_{\mathbf{y}_{i}}$ as follows.

$$
S S\left(\mathbf{X}_{W}, \mathbf{Y}_{W}\right)=\frac{\sum_{i=1}^{n} 1-\left|M_{\mathbf{x}_{i}}-M_{\mathbf{y}_{i}}\right|}{n} .
$$

\subsection{Luminance}

To compare image luminance we propose to use spherical coordinates computed from RGB values [23]. Luminance correspond with the radius parameter given by

$$
L \mathbf{x}_{i}=\sqrt{\mathbf{x}_{i}(1)^{2}+\mathbf{x}_{i}(2)^{2}+\mathbf{x}_{i}(3)^{2}}
$$

The luminance similarity between $\mathbf{X}_{W}$ and $\mathbf{Y}_{W}$ is obtained through the corresponding expression in [13] as 


$$
S L\left(\mathbf{X}_{W}, \mathbf{Y}_{W}\right)=\frac{2 \overline{L_{\mathbf{X}_{W}} L_{\mathbf{Y}_{W}}}}{\overline{L_{\mathbf{X}_{W}}}+\bar{L}_{\mathbf{Y}_{W}}}
$$

where $\overline{L_{\mathbf{X}_{W}}}$ and $\overline{L_{\mathbf{Y}_{W}}}$ are the mean luminance in each patch. In the case that $\overline{L_{\mathbf{X}_{W}}}=\overline{L_{\mathbf{Y}_{W}}}=0$ we $\operatorname{assign} S L\left(\mathbf{X}_{W}, \mathbf{Y}_{W}\right)=1$.

Finally, the similarity between $\mathbf{X}_{W}$ and $\mathbf{Y}_{W}$ results from combining the three previous measures as follows

$$
S\left(\mathbf{X}_{W}, \mathbf{Y}_{W}\right)=S C\left(\mathbf{X}_{W}, \mathbf{Y}_{W}\right)^{\alpha} \cdot S S\left(\mathbf{X}_{W}, \mathbf{Y}_{W}\right)^{\beta} \cdot S L\left(\mathbf{X}_{W}, \mathbf{Y}_{W}\right)^{\gamma}
$$

where $\alpha, \beta, \gamma>0$ are parameters used to adjust relative importance of three

components. As commented above, the average of all $S\left(\mathbf{X}_{W}, \mathbf{Y}_{W}\right)$ provides the similarity between $\mathbf{X}$ and $\mathbf{Y}$, that will be high only if the three similarities are high.

Finally, we would like to point out that in each processing patch the number of operations is proportional to the number of pixels, so for the whole method we have also a linear computational cost.

\section{Experimental study}

In order to study the performance of our proposal and also to compare with other approaches we make a comparison with respect to a survey of perceptual observations as follows.

We have chosen the four color bmp images in Figure 1: Goldhill, Lenna, 
Baboon, and Parrots. To better appreciate low resolution differences we have taken a small part of $68 \times 68$ pixels of the original images. We have applied a series of 10 different distortions to each of the test images. The distortions applied over the image Parrots along with the software use in each case, which are shown in Figure 2, are the following.

1. jpg compression of ratio 20\% (MS Picture Manager)

2. Increase brightness by $15 \%$ (MS Picture Manager)

3. Increase contrast by $15 \%$ (MS Picture Manager)

4. Gaussian blur with radius 1.5 (Corel Draw X5)

5. Addition of $5 \%$ of impulsive noise (imnoise function from Matlab)

6. Addition of white Gaussian noise with standard deviation equals to $10 \%$ of the maximum value in the channels (imnoise function from Matlab)

7. Filtering of original image with [24]

8. Addition of Gaussian noise as in 6) and filtering with [24]

9. Filtering of original image with Vector Median Filter (VMF) [25]

10. Addition of $5 \%$ of impulsive noise as in 5) and filtering with Vector Median Filter (VMF) [25]

In the survey, we asked independent observers to rank the 10 distorted images with respect to its similarity to the original image (1st the most similar, 10th the least). We did this through a questionnaire available on the internet address [27] to get as many answers as possible. We received 108 complete answers. We processed them to remove outliers using boxplot 
and we found 4 outliers that could be due to the observer not paying enough attention or to wrong understanding. Finally, we average the ranks obtained by each of the distorted images and we re-scale the average rankings to the interval $[1,10]$.

Next, we measure the similarity between all distorted images and the original one with the usual similarity measures MAE, MSE, NCD, as well as with Structural Similarity Index (SSIM) $[13,14]$ (used by averaging after component-wise application in each channel), FSIMc [26], CMSSIM [16] and the proposed method (Fuzzy Color Structural Similarity, FCSS). To assess the match between these measures and the survey perceptual observations, we re-scaled similarity measures results to the interval $[1,10]$. In this way we can measure the similarity between each measure ranking and the perceptual ranking.

For our proposal we try different parameter settings and one providing a nice overall performance is the following: $t=256$, patch size $q=4$ and $\alpha=\beta=\gamma=1$.

Tables 1 - 8 show the ranks obtained in our survey for each image and those provided by the methods in the comparison. To measure the match between perceptual observations and the similarity measures we computed the Root Mean Squared Error (RMSE) [1] and the correlation coefficient $r$ between the re-scaled ranks of each similarity measure and the re-scaled ranks of the visual observations.

From these results we can see that performance of SSIM, FSIMc and FCSS 
is much better than the rest of the methods. CMSSIM only works well for Goldhill image, which suggests that it is too sensitive to the image features. SSIM exhibits a very high performance $(r>0.9)$ in two cases (Goldhill and Lenna) but much lower $(r<0.8)$ in another two cases (Baboon and Parrots). FSIMc performs very well for GoldHill and Lenna $(r>0.9)$, well for Parrots $(r \sim 0.8)$, but worse for Baboon image, where its performance drops with respect to FCSS $(r<0.8)$. On the other hand, FCSS exhibits a consistent high performance in all cases $(r \in[0.80,0.90])$ and it is better than SSIM for Baboon and Parrots images and better than FSIMc for Baboon image.

In order to understand these pretty high differences in the performance of SSIM and FSIMc for different images we analyzed several features of the images and we realized that there is significant differences with respect to their correlations among the image channels. These correlations are shown in Table 9. We see that correlations in Goldhill and Lenna images are high in all cases, whereas in Parrots and Baboon appear some medium and low correlations respectively. This implies that SSIM is only able to provide high performance when the correlation among the color channels is high in all cases. However, when for a couple of channels the correlation is not high, SSIM performs worse. This is most probably due to the component-wise application of SSIM. FSIMc performs better from this point of view and still performs well in the presence of some medium correlations (Parrots), but its performance drops for the Baboon image were the correlation between the $\mathrm{R}$ and $\mathrm{B}$ channels is very low and the rest are not high. We see that FSIMc 
is sensitive to low correlations between channels which probably means that its capability to take into account correlation can be improved. On the other hand, FCSS performance is independent from the correlation among the image channels which in turns indicates proper correlation management. This is interesting for practical applications and also for possible adaptations to other types of multichannel images and future research.

These results justify the need of keeping active the research on specific methods for color image similarity.

\section{Conclusions}

In this paper we have proposed a method to measure the similarity between two color images that uses fuzzy metrics. The similarity between the images takes into account three factors: structural similarity, contrast similarity, and luminance similarity. The method takes into account the correlation among the image channels by processing the images as vector fields. Experimental results employing a survey of observations show that the global performance of our proposal is competitive with best state of the art methods and that it shows some advantages in performance for images with low correlation among some image channels, which is interesting for future research. 


\section{Acknowledgements}

S. Komara acknowledges a partial financial support by the ESF project 2009/0162/1DP/1.1.2.1.1/09/IPIA/VIAA/004.

\section{References}

[1] K.N. Plataniotis, A.N. Venetsanopoulos, Color Image Processing and Applications, Springer-Verlag, (2000) 355 pp 1-45, 51-100, 109-157.

[2] Z. Wang, A.C. Bovik, Mean Squared Error: Love It or Leave It?,IEEE Signal Processing Magazine 26(1)(2009)98-117.

[3] T. Mitsa, K. Varkur, Evaluation of contrast sensitivity functions for the formulation of quality measures incorporated in halftoning algorithms, IEEE International Conference on Acoustic, Speech and Signal Processing 5(1993)301-304.

[4] I. Avcibas, B. Sankur, K. Sayood, Statistical evaluation of image quality measures, Journal of Electronic Imaging 11(2)(2002)206-223.

[5] A.M. Eskicioglu, P.S. Fisher, Image quality measures and their performance, IEEE Trans. Communications 43(12)(1995)2959-2965.

[6] S. Susstrunk, S. Winkler, Color image quality on the internet, Internet Imaging V 5304(2004) 118-131. 
[7] C. Charrier, T. Eude, A psychovisual color image quality metric integrating both intra and inter channel masking effect, European Signal Processing Conf., (2004).

[8] P. Le Callet, D. Barba, A robust quality metric for color image quality assessment, IEEE International Conference on Image Processing (2003)437440.

[9] Z. Wang, G. Wu, H.R. Sheikh, E.P. Simoncelli, E.-H. Yang, A.C. Bovik, Quality-aware images, IEEE Trans. Image Processing 15(6)(2006)16801689.

[10] A. Ninassi, P. Le Callet, F. Autrusseau, Pseudo no reference image quality metric using perceptual data hiding, Proc. SPIE Conf. Human Vision and Electronic Imaging XI 6057(2006).

[11] S.M. Lee, J.H. Xin, S. Westland, Evaluation of image similarity by histogram intersection, Color Research \& Application 30(4)(2005)265-274.

[12] K. Roy, J. Mukherjee, Image Similarity Measure using Color Histogram, Color Coherence Vector, and Sobel Method, International Journal of Science and Research 2(1)(2013)538-543.

[13] Z.Wang, A.C. Bovik, A Universal Image Quality Index, IEEE Signal Processing Letters 9(3)(2002)81-84.

[14] Z. Wang, A.C. Bovik, H.R. Sheikh, E.P. Simoncelli, Image Quality As- 
sessment: From Error Visibility to Structural Similarity, IEEE Transactions on Image Processing 13(4)(2004)600-612.

[15] Z. Wang, E.P. Simoncelli, A.C. Bovik, Multiscale structural similarity for image quality assessment, Proc. of the 37th IEEE Asilomar Conference on Signals, Systems and Computers 2(2003)1398-1402. Source code is available at http://sse.tongji.edu.cn/linzhang/IQA/Evalution_ MS_SSIM/eva-MS-SSIM.htm.

[16] M. Hassan, C. Bhagvati, Structural Similarity Measure for Color Images, International Journal of Computer Applications 43(14)(2012)7-12.

[17] C. Li, A.C. Bovik, Content-partitioned structural similarity index for image quality assessment, Signal Processing: Image Communication 25 (2010)517-526.

[18] D.M. Chandler, S.S. Hemami, VSNR: A Wavelet base Visual Signal-toNoise Ratio for Natural Images, IEEE Transaction on Image Processing, 16(9)(2007)2284-2298.

[19] S. Morillas, V. Gregori, G. Peris-Fajanes, P. Latorre, A fast impulsive noise color image filter using fuzzy metrics, Real-Time Imaging 11(56)(2005)417-428.

[20] S. Morillas, V. Gregori, G. Peris-Fajanes, P. Latorre, A new vector median filter based on fuzzy metrics, Lecture Notes in Computer Science 3656,(2005) pp. 81-90. 
[21] S. Morillas, V. Gregori, A. Sapena, Fuzzy bilateral filtering for color images, Lecture Notes in Computer Science 4141, (2006) pp. 138-145.

[22] J.G. Camarena, V. Gregori, S. Morillas, A. Sapena, Two-step fuzzy logic-based method for impulse noise detection in color images, Pattern Recognition Letters 31(13)(2010) 1842-1849.

[23] E.S. Hore, B. Qiu, H.R. Wu, Noise estimation in spherical coordinates for color image restoration, Optical Engineering 44(4)(2005).

[24] S. Morillas, V. Gregori, A. Hervás, Fuzzy peer groups for reducing mixed gaussian-impulse noise from color images, IEEE Trans. Image Processing $18(7)(2009)$ 1452-1466.

[25] J. Astola, P. Haavisto, Y. Neuvo, Vector Median Filters, Proceedings of the IEEE 78(4)(1990)678-689.

[26] Lin Zhang, Lei Zhang, X. Mou, D. Zhang, FSIM: A Feature Similarity Index for Image Quality Assessment, IEEE Trans. Image Processing 20(8)(2011) 2378-2386. Source code is available at http://www4.comp. polyu.edu.hk/ cslzhang/IQA/FSIM/FSIM.htm.

[27] http://www . esurveyspro.com/Survey . aspx?id= $8 a 99 e e c 7-d a 6 c-4691-9 b 40-1 a 2 e 4 d a 3 d 023$ 


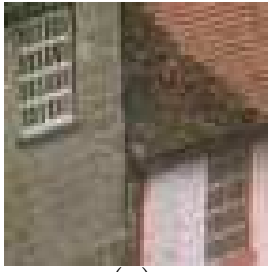

(a)

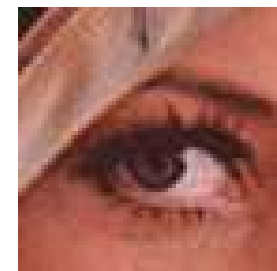

(b)

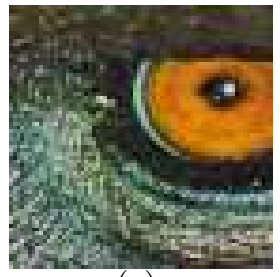

(c)

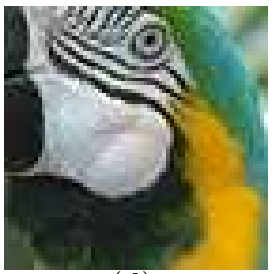

(d)

Figure 1: Images for tests: (a) Goldhill, (b) Lenna, (c) Baboon, and (d) Parrots. 


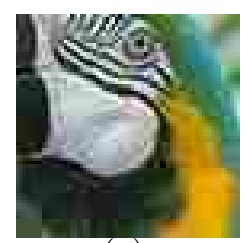

(a)

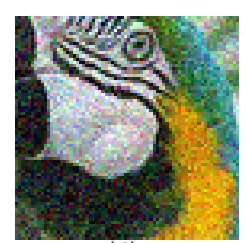

(f)

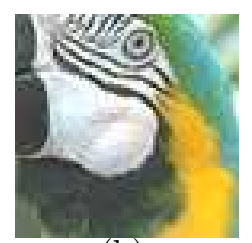

(b)

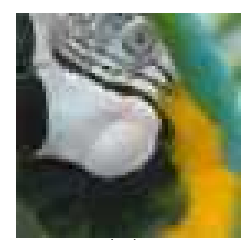

(g)

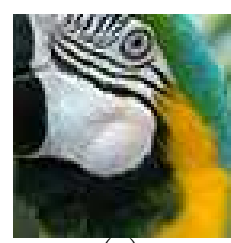

(c)

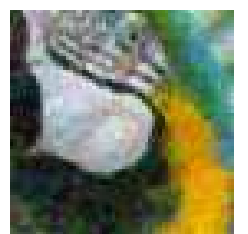

(h)

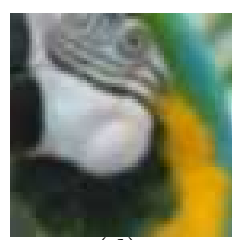

(d)

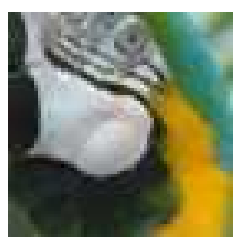

(i)

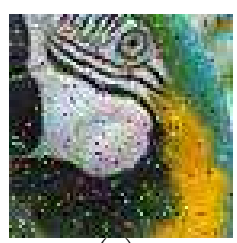

(e)

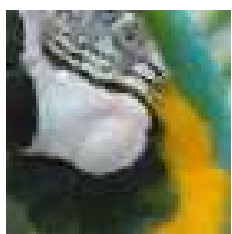

(j)

Figure 2: Distortions applied to the image Parrots: (a) jpg compression of ratio 20\% (MS Picture Manager), (b) Increase brightness by 15\% (MS Picture Manager), (c) Increase contrast by $15 \%$ (MS Picture Manager), (d) Gaussian blur with radius 1.5 (Corel Draw X5), (e) Addition of 5\% of impulsive noise (Matlab according to [1]), (f) Addition of white Gaussian noise with standard deviation equals to $10 \%$ of the maximum value in the channels (Matlab according to [1]), (g) Filtering of original image with [24], (h) Addition of Gaussian noise as in (f) and filtering with [24], (i) Filtering of original image with Vector Median Filter (VMF) [25], (j) Addition of $5 \%$ of impulsive noise as in (e) and filtering with Vector Median Filter (VMF) [25]. 
Table 1: Performance comparison for Goldhill image. * denotes re-scaled ranking/similarity to the interval $[1,10]$. RMSE and $r$ denote the Root Mean Squared Error and correlation coefficient, respectively, between the re-scaled ranks of each similarity measure and the re-scaled ranks of the visual observations.

\begin{tabular}{ccccccccc}
\hline Effect & Survey & Survey $^{*}$ & MAE & MAE* $^{*}$ & MSE & MSE* & SSIM & SSIM $^{*}$ \\
\hline 1 & 5.952 & 7.03 & 10.677 & 2.41 & 196.675 & 2.01 & 0.638 & 6.75 \\
2 & 2.615 & 2.93 & 37.169 & 10.00 & 1382.581 & 10.00 & 0.949 & 1.64 \\
3 & 1.038 & 1.00 & 5.754 & 1.00 & 47.118 & 1.00 & 0.988 & 1.00 \\
4 & 6.212 & 7.35 & 8.242 & 1.71 & 129.801 & 1.56 & 0.741 & 5.05 \\
5 & 8.375 & 10.00 & 6.798 & 1.30 & 958.967 & 7.15 & 0.468 & 9.53 \\
6 & 8.192 & 9.78 & 20.326 & 5.17 & 646.569 & 5.04 & 0.447 & 9.88 \\
7 & 4.596 & 5.36 & 8.017 & 1.65 & 166.062 & 1.80 & 0.692 & 5.86 \\
8 & 8.279 & 9.88 & 15.341 & 3.75 & 389.531 & 3.31 & 0.440 & 10.00 \\
9 & 4.596 & 5.36 & 8.129 & 1.68 & 164.759 & 1.79 & 0.687 & 5.93 \\
10 & 5.144 & 6.04 & 8.553 & 1.80 & 178.725 & 1.89 & 0.675 & 6.13 \\
\hline RMSE & & 0 & & 5.318 & & 4.744 & & 0.884 \\
r & & 1 & -0.108 & & 0.124 & & 0.960 \\
\hline
\end{tabular}

Table 2: Performance comparison for Goldhill image

\begin{tabular}{ccccccccccc}
\hline Effect & Survey & Survey $^{*}$ & NCD & NCD & CMSSIM & CMSSIM $^{*}$ & FSIMc & FSIMc $^{*}$ & FCSS $^{\text {FCSS }}$ \\
\hline 1 & 5.952 & 7.03 & 0.071 & 2.58 & 0.654 & 5.64 & 0.889 & 7.31 & 0.895 & 4.58 \\
2 & 2.615 & 2.93 & 0.130 & 5.16 & 0.923 & 1.24 & 0.995 & 1.00 & 0.931 & 2.62 \\
3 & 1.038 & 1.00 & 0.036 & 1.00 & 0.920 & 1.29 & 0.993 & 1.09 & 0.961 & 1.00 \\
4 & 6.212 & 7.35 & 0.045 & 1.41 & 0.937 & 1.00 & 0.877 & 8.03 & 0.872 & 5.86 \\
5 & 8.375 & 10.00 & 0.120 & 4.72 & 0.489 & 8.33 & 0.863 & 8.81 & 0.796 & 10.00 \\
6 & 8.192 & 9.78 & 0.239 & 10.00 & 0.447 & 9.02 & 0.843 & 10.00 & 0.829 & 8.20 \\
7 & 4.596 & 5.36 & 0.046 & 1.48 & 0.925 & 1.20 & 0.911 & 5.96 & 0.896 & 4.52 \\
8 & 8.279 & 9.88 & 0.149 & 5.99 & 0.387 & 10.00 & 0.857 & 9.19 & 0.888 & 4.96 \\
9 & 4.596 & 5.36 & 0.046 & 1.45 & 0.927 & 1.16 & 0.908 & 6.15 & 0.895 & 4.57 \\
10 & 5.144 & 6.04 & 0.049 & 1.59 & 0.921 & 1.27 & 0.906 & 6.29 & 0.897 & 4.46 \\
\hline RMSE & \multicolumn{3}{c}{3.916} & & 3.260 & & 0.853 & 0.958 & 0.971 \\
r & 0 & 1 & 0.574 & & 0.805 & & 0.873 \\
\hline
\end{tabular}


Table 3: Performance comparison for Lenna image

\begin{tabular}{|c|c|c|c|c|c|c|c|c|}
\hline Effect & Survey & Survey* & MAE & MAE* $^{*}$ & MSE & MSE* $^{*}$ & SSIM & SSIM* $^{*}$ \\
\hline 1 & 6.423 & 6.88 & 8.864 & 2.35 & 140.249 & 1.66 & 0.755 & 4.57 \\
\hline 2 & 4.337 & 4.49 & 37.396 & 10.00 & 1398.719 & 10.00 & 0.930 & 1.73 \\
\hline 3 & 1.288 & 1.00 & 7.229 & 1.92 & 69.314 & 1.19 & 0.975 & 1.00 \\
\hline 4 & 5.731 & 6.08 & 5.319 & 1.40 & 60.199 & 1.13 & 0.878 & 2.58 \\
\hline 5 & 9.154 & 10.00 & 6.266 & 1.66 & 937.370 & 6.94 & 0.456 & 9.44 \\
\hline 6 & 8.788 & 9.58 & 20.248 & 5.41 & 641.076 & 4.98 & 0.422 & 10.00 \\
\hline 7 & 3.279 & 3.28 & 3.809 & 1.00 & 40.924 & 1.00 & 0.894 & 2.32 \\
\hline 8 & 8.327 & 9.05 & 12.938 & 3.45 & 266.176 & 2.49 & 0.560 & 7.75 \\
\hline 9 & 3.240 & 3.23 & 3.902 & 1.02 & 40.890 & 1.00 & 0.891 & 2.36 \\
\hline 10 & 4.433 & 4.60 & 4.436 & 1.17 & 52.697 & 1.08 & 0.872 & 2.67 \\
\hline RMSE & & 0 & & 4.624 & & 4.223 & & 1.811 \\
\hline $\mathrm{r}$ & & 1 & & 0.157 & & 0.377 & & 0.930 \\
\hline
\end{tabular}

Table 4: Performance comparison for Lenna image

\begin{tabular}{ccccccccccc}
\hline Effect & Survey & Survey $^{*}$ & NCD & NCD* & CMSSIM & CMSSIM $^{*}$ & FSIMc & FSIMc* & FCSS & FCSS* \\
\hline 1 & 6.423 & 6.88 & 0.084 & 3.07 & 0.544 & 7.56 & 0.907 & 5.42 & 0.925 & 2.88 \\
2 & 4.337 & 4.49 & 0.148 & 5.46 & 0.702 & 8.97 & 0.997 & 1.00 & 0.934 & 2.44 \\
3 & 1.288 & 1.00 & 0.072 & 2.61 & 0.671 & 10.00 & 0.993 & 1.18 & 0.963 & 1.00 \\
4 & 5.731 & 6.08 & 0.037 & 1.30 & 0.922 & 1.17 & 0.926 & 4.46 & 0.925 & 2.88 \\
5 & 9.154 & 10.00 & 0.110 & 4.06 & 0.531 & 8.07 & 0.841 & 8.62 & 0.778 & 10.00 \\
6 & 8.788 & 9.58 & 0.269 & 10.00 & 0.428 & 9.84 & 0.813 & 10.00 & 0.813 & 8.32 \\
7 & 3.279 & 3.28 & 0.030 & 1.03 & 0.955 & 1.01 & 0.958 & 2.91 & 0.943 & 1.97 \\
8 & 8.327 & 9.05 & 0.159 & 5.88 & 0.399 & 9.82 & 0.869 & 7.29 & 0.901 & 4.02 \\
9 & 3.240 & 3.23 & 0.029 & 1.00 & 0.958 & 1.00 & 0.957 & 2.97 & 0.943 & 1.98 \\
10 & 4.433 & 4.60 & 0.032 & 1.14 & 0.951 & 1.15 & 0.948 & 3.40 & 0.939 & 2.18 \\
\hline RMSE & & 0 & & 3.292 & & 3.900 & & 1.543 & 0.929 & 2.578 \\
r & & & 0.640 & & 0.386 & & & 0.850 \\
\hline
\end{tabular}

Table 5: Performance comparison for Baboon image

\begin{tabular}{ccccccccc}
\hline Effect & Survey & Survey $^{*}$ & MAE & MAE $^{*}$ & MSE & MSE $^{*}$ & SSIM & SSIM $^{*}$ \\
\hline 1 & 5.615 & 6.20 & 17.536 & 4.19 & 523.011 & 3.94 & 0.599 & 7.81 \\
2 & 3.077 & 3.30 & 37.451 & 10.00 & 1403.372 & 10.00 & 0.894 & 1.88 \\
3 & 1.067 & 1.00 & 8.603 & 1.59 & 96.246 & 1.00 & 0.938 & 1.00 \\
4 & 7.519 & 8.38 & 14.082 & 3.18 & 376.375 & 2.93 & 0.665 & 6.49 \\
5 & 8.933 & 10.00 & 6.596 & 1.00 & 1059.051 & 7.63 & 0.616 & 7.48 \\
6 & 6.510 & 7.23 & 19.657 & 4.81 & 604.993 & 4.50 & 0.648 & 6.83 \\
7 & 5.404 & 5.96 & 13.289 & 2.95 & 433.458 & 3.32 & 0.637 & 7.06 \\
8 & 6.577 & 7.30 & 19.487 & 4.76 & 665.686 & 4.92 & 0.490 & 10.00 \\
9 & 4.952 & 5.45 & 13.390 & 2.98 & 433.040 & 3.32 & 0.639 & 7.02 \\
10 & 5.346 & 5.90 & 14.103 & 3.19 & 474.321 & 3.60 & 0.618 & 7.43 \\
\hline RMSE & 0 & & 4.383 & & 3.395 & & 1.673 \\
r & 1 & -0.271 & & 0.217 & & 0.783 \\
\hline
\end{tabular}


Table 6: Performance comparison for Baboon image

\begin{tabular}{|c|c|c|c|c|c|c|c|c|c|c|}
\hline Effect & Survey & Survey* & NCD & $\mathrm{NCD}^{*}$ & CMSSIM & CMSSIM* & FSIMc & FSIMc $^{*}$ & FCSS & FCSS* \\
\hline 1 & 5.615 & 6.20 & 0.205 & 5.26 & 0.363 & 9.52 & 0.887 & 6.68 & 0.864 & 5.89 \\
\hline 2 & 3.077 & 3.30 & 0.189 & 4.61 & 0.729 & 2.72 & 0.994 & 1.00 & 0.893 & 3.83 \\
\hline 3 & 1.067 & 1.00 & 0.103 & 1.00 & 0.696 & 3.35 & 0.991 & 1.17 & 0.934 & 1.00 \\
\hline 4 & 7.519 & 8.38 & 0.127 & 1.99 & 0.822 & 1.00 & 0.824 & 10.00 & 0.805 & 10.00 \\
\hline 5 & 8.933 & 10.00 & 0.128 & 2.07 & 0.470 & 7.53 & 0.887 & 6.66 & 0.841 & 7.51 \\
\hline 6 & 6.510 & 7.23 & 0.319 & 10.00 & 0.400 & 8.84 & 0.874 & 7.37 & 0.851 & 6.79 \\
\hline 7 & 5.404 & 5.96 & 0.130 & 2.14 & 0.770 & 1.97 & 0.868 & 7.67 & 0.840 & 7.54 \\
\hline 8 & 6.577 & 7.30 & 0.243 & 6.84 & 0.338 & 10.00 & 0.837 & 9.34 & 0.836 & 7.82 \\
\hline 9 & 4.952 & 5.45 & 0.128 & 2.06 & 0.801 & 1.39 & 0.867 & 7.72 & 0.843 & 7.36 \\
\hline 10 & 5.346 & 5.90 & 0.135 & 2.37 & 0.749 & 2.36 & 0.866 & 7.77 & 0.843 & 7.32 \\
\hline RMSE & & 0 & & 3.907 & & 3.64 & & 1.874 & & 1.337 \\
\hline $\mathrm{r}$ & & 1 & & 0.211 & & 0.357 & & 0.782 & & 0.859 \\
\hline
\end{tabular}

Table 7: Performance comparison for Parrots image

\begin{tabular}{|c|c|c|c|c|c|c|c|c|}
\hline Effect & Survey & Survey* & MAE & MAE* & MSE & MSE* & SSIM & SSIM* \\
\hline 1 & 5.240 & 6.15 & 13.044 & 2.91 & 346.224 & 2.65 & 0.749 & 5.52 \\
\hline 2 & 2.663 & 2.99 & 37.285 & 10.00 & 1395.746 & 10.00 & 0.886 & 2.19 \\
\hline 3 & 1.038 & 1.00 & 9.179 & 1.78 & 109.858 & 1.00 & 0.935 & 1.00 \\
\hline 4 & 6.865 & 8.15 & 11.966 & 2.60 & 443.218 & 3.33 & 0.809 & 4.06 \\
\hline 5 & 8.029 & 9.58 & 6.503 & 1.00 & 1098.513 & 7.92 & 0.585 & 9.49 \\
\hline 6 & 6.240 & 7.38 & 19.517 & 4.81 & 595.748 & 4.40 & 0.564 & 10.00 \\
\hline 7 & 5.192 & 6.10 & 7.515 & 1.30 & 286.272 & 2.23 & 0.876 & 2.42 \\
\hline 8 & 8.375 & 10.00 & 16.690 & 3.98 & 569.456 & 4.22 & 0.613 & 8.80 \\
\hline 9 & 5.115 & 6.00 & 7.670 & 1.34 & 291.128 & 2.27 & 0.874 & 2.46 \\
\hline 10 & 6.240 & 7.38 & 8.651 & 1.63 & 328.090 & 2.53 & 0.857 & 2.89 \\
\hline RMSE & & 0 & & 5.341 & & 4.266 & & 2.688 \\
\hline $\mathrm{r}$ & & 1 & & -0.285 & & 0.118 & & 0.739 \\
\hline
\end{tabular}

Table 8: Performance comparison for Parrots image

\begin{tabular}{|c|c|c|c|c|c|c|c|c|c|c|}
\hline Effect & Survey & Survey* & NCD & $\mathrm{NCD}^{*}$ & CMSSIM & CMSSIM* & FSIMc & FSIMc* & FCSS & FCSS* \\
\hline 1 & 5.240 & 6.15 & 0.115 & 3.71 & 0.544 & 7.67 & 0.894 & 6.14 & 0.887 & 3.73 \\
\hline 2 & 2.663 & 2.99 & 0.175 & 5.94 & 0.702 & 5.12 & 0.989 & 1.12 & 0.890 & 3.49 \\
\hline 3 & 1.038 & 1.00 & 0.088 & 2.71 & 0.671 & 5.62 & 0.991 & 1.00 & 0.924 & 1.00 \\
\hline 4 & 6.865 & 8.15 & 0.060 & 1.66 & 0.922 & 1.58 & 0.905 & 5.53 & 0.849 & 6.53 \\
\hline 5 & 8.029 & 9.58 & 0.112 & 3.60 & 0.531 & 7.88 & 0.838 & 9.12 & 0.802 & 10.00 \\
\hline 6 & 6.240 & 7.38 & 0.284 & 10.00 & 0.428 & 9.53 & 0.821 & 10.00 & 0.832 & 7.82 \\
\hline 7 & 5.192 & 6.10 & 0.042 & 1.00 & 0.955 & 1.06 & 0.950 & 3.14 & 0.898 & 2.95 \\
\hline 8 & 8.375 & 10.00 & 0.187 & 6.41 & 0.399 & 10.00 & 0.862 & 7.82 & 0.843 & 6.94 \\
\hline 9 & 5.115 & 6.00 & 0.042 & 1.01 & 0.958 & 1.00 & 0.948 & 3.27 & 0.895 & 3.14 \\
\hline 10 & 6.240 & 7.38 & 0.047 & 1.20 & 0.951 & 1.12 & 0.939 & 3.75 & 0.891 & 3.44 \\
\hline RMSE & & 0 & & 4.518 & & 4.104 & & 2.270 & & 2.283 \\
\hline$r$ & & 1 & & 0.125 & & 0.221 & & 0.804 & & 0.801 \\
\hline
\end{tabular}

Table 9: Correlation in image channels

\begin{tabular}{ccccc}
\hline Channels & Goldhill & Lenna & Baboon & Parrots \\
\hline RG & 0.92 & 0.89 & 0.69 & 0.9 \\
RB & 0.89 & 0.78 & 0.1 & 0.5 \\
GB & 0.97 & 0.96 & 0.7 & 0.75 \\
\hline
\end{tabular}

